\title{
Guarding Orthogonal Galleries with Rectangular Rooms
}

\author{
Antonio L. Bajuelos ${ }^{1}$, Sergey Bereg ${ }^{2}$ And Mafalda Martins ${ }^{1}$ \\ ${ }^{1}$ Universidade de Aveiro and CIDMA, Portugal \\ ${ }^{2}$ Department of Computer Science, University of Texas at Dallas, Box 830688, Richardson, \\ TX 75083 USA \\ Email: besp@utdallas.edu,\{leslie,mafalda.martins\}@ua.pt
}

\begin{abstract}
Consider an orthogonal art gallery partitioned into $n$ rectangular rooms. If two rooms are adjacent there is a door connecting them and a guard positioned at this door will see both rooms. In [2] it is shown that any rectangular gallery can be guarded with $\lceil n / 2\rceil$ guards. We prove that the same bound holds for L-shape polygons. We extend it to staircases and prove that an orthogonal staircase with $n$ rooms and $r$ reflex vertices can be guarded with $\lceil(n+\lfloor r / 2\rfloor) / 2\rceil$ guards. Then we prove an upper bound on the number of guards for arbitrary orthogonal polygon with orthogonal holes. This result improves the previous bound by Czyzowicz et al [2] (even in the case of polygon without holes).
\end{abstract}

Keywords: Art Gallery Problem; Computational Geometry

Received 00 January 2009; revised 00 Month 2009

\section{INTRODUCTION}

The Art Gallery Problem was posed by Viktor Klee in 1973: "How many guards are always sufficient to guard any simple polygon $P$ with $v$ vertices?"3 Chvátal has shown that $\lfloor v / 3\rfloor$ guards are always sufficient and sometimes necessary to cover $P$ [1]. This problem has grown into a field in computational geometry and computer science. Many variants of art gallery problems have been studied since. They have applications in sensor networks, computer vision, robotics, motion planning, and computer-aided design, see for example [6]. For a general survey on art gallery problems see, e.g., $[8,10]$.

Orthogonal (rectilinear) polygons are polygons whose edges are parallel to the coordinate axes. This subclass of polygons is important because real life buildings are orthogonal. Furthermore, orthogonal polygons arise naturally in many applications, for example VLSI design and computer graphics. The main result for this class of polygons is by Kahn et al [4] that $\lfloor v / 4\rfloor$ guards are always sufficient and occasionally necessary to guard an orthogonal polygon with $v$ vertices.

Czyzowicz et al [2] studied the following variant of Art Gallery Problem. Suppose that have a rectangular gallery, subdivided into $n$ rectangular rooms. Suppose that any two adjacent rooms have a door connecting them. How many guards need to be stationed in the gallery to cover all the rooms? Observe that if a

\footnotetext{
${ }^{3}$ By simple polygon we mean a region of the plane enclosed by a simple cycle of line segments and its interior is topologically equivalent to a disk.
}

guard is positioned at a door, he will see two adjacent rooms. Their main result is that $\lceil n / 2\rceil$ guards are always sufficient to cover all the rooms.

An orthogonal polygon with holes is defined as $P \backslash$ $\cup_{i=1}^{k} P_{i}$ where $P$ and $P_{i}$ for all $i$ are orthogonal polygons such that (i) polygons $P_{i}$ are in the interior of $P$ and (ii) polygons $P_{i}$ are pairwise disjoint. Czyzowicz et al [2] also proved that an orthogonal polygon with $v$ vertices, $h$ holes and $n$ rectangular rooms can be always covered with $\lceil(2 n+v-2 h-4) / 4\rceil$ guards. This bound can be written as $\lceil(n+r-3 h) / 2\rceil$ where $r$ is the number of reflex vertices of the orthogonal gallery (a vertex of a polygon is called reflex if the interior angle between its two incident edges is greater than $\pi$. To verify that the two bounds are equal one can use formula $v^{\prime}=2 r^{\prime}+4$ [7] for any orthogonal polygon (without holes) with $v^{\prime}$ vertices and $r^{\prime}$ reflex vertices. The formula can be used for $P$ and all $P_{i}$.

In our work we focus on problems of guarding orthogonal galleries with rectangular rooms. Our results improve some bounds in [2]. First, we prove $\lceil n / 2\rceil$ bound for guarding a gallery of L-shape with $n$ rooms. The previous bound is $\lceil(n+1) / 2\rceil[2]$.

Second, we prove that an orthogonal staircase with $n$ rooms and $r$ reflex vertices can be guarded with $\lceil(n+\lfloor r / 2\rfloor) / 2\rceil$ guards. We also show that, for any $r>1$, there are examples of orthogonal staircase galleries that require this number of guards.

Third, we prove an upper bound on the number of guards for arbitrary orthogonal polygon with holes, see Theorem 3.1. There are two cases in the theorem and 
the general upper bound can be expressed as $\lceil(n+r-$ $\left.\left.3 h-k_{1}\right) / 2\right\rceil$ where $k_{1}$ informally is the the number of pockets of $P$ that have horizontal or vertical lid. This result improves the previous bound $\lceil(n+r-3 h) / 2\rceil$ on the number of guards [2].

\section{ORTHOGONAL STAIRCASES}

An alternating subchain of an orthogonal polygon is a subchain with at least one internal vertex, with the first and last internal vertices being convex, and with consecutive internal vertices alternating between convex and reflex. A staircase polygon is an orthogonal polygon consisting of three consecutive convex vertices $v_{1}$ (top-left vertex), $v_{2}$ (down-left vertex) and $v_{3}$ (downright vertex) and vertices $v_{1}$ and $v_{3}$ connected by one alternating subchain.

We start with a simple staircase polygon that contains only one reflex vertex. We call these polygons L-shaped polygons.

LEMMA 2.1. L-shaped polygon with $n$ rooms can be guarded with $\lceil n / 2\rceil$ guards.

Proof. Our proof uses similar ideas of Theorem on rectangular galleries [2]. We form a rectangle by taking a mirror image of the L-shaped polygon and adding 2 connecting rooms, see Figure 1 (a). Take 5 copies of $R$ and complete a new rectangle as shown in Figure 1 (b). The dual graph $G$, including the outer face, is planar and 4-connected by Theorem 2.3 from [2]. It has a Hamiltonian curcuit by a theorem of Tutte [9].

It follows easily that, for every Hamiltonian curcuit $C$ in $G$, there must exists a region $D=A \cup R^{\prime} \cup B$ formed by $R^{\prime}$, a copy of $R$ and two side rectangles $A$ and $B$ of $R^{\prime}$ such that $C$ visits $D$ in the order $A-R^{\prime}-B$ or $B-R^{\prime}-A$ (because $C$ passes through the top/bottom rectangle using at most two copies of $R$ ).

It is not hard to see that circuit $C$ visits one of the L-shaped polygons of $R^{\prime}$ exactly once, see Figure 1 (c). Using every other edge of $C$ passing through $R^{\prime}$ gives us $\lfloor n / 2\rfloor$ of the dual graph of $R^{\prime}$. We place a guard for each of these edges. If $n$ is odd then we place a guard for the remaining vertex. This implies the bound.

It is easy to see that bound $\lceil n / 2\rceil$ does not hold for staircases with $r>1$, see Figure 2 for an example. Czyzowicz et al [2] proved that a staircase polygon with $r$ reflex vertices can be guarded with $\lceil(n+r) / 2\rceil$ guards. This bound can be improved using Lemma 1 (if $n+r$ is odd).

Corollary 2.1. If $r>1$ then any staircase with $r$ reflex vertices can be guarded with $\lceil(n+r-1) / 2\rceil$.

Proof. If $r>1$ then add $r-1$ rooms to staircase so as to obtain L-shape, see Figure 3. The L-shape can be guarded by $\lceil((n+r-1) / 2\rceil$ guards by Lemma 1 . Our next observation is essential to our proof: a guard covering new rooms may be (i) between two new rooms

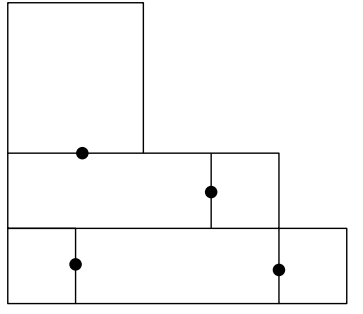

(a)

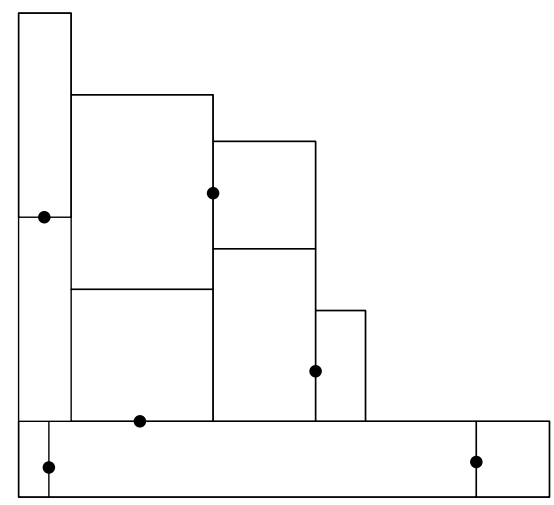

(b)

FIGURE 2. (a) A staircase polygon with 6 rooms that requires 4 guards. (b) A staircase polygon with 10 rooms that requires 6 guards.

or (ii) between a new room and an adjacent room from the staircase polygon. In the first case this guard is not necessary for the original staircase and may be removed. In the second case this guard may placed in another door of the room of the original staircase.

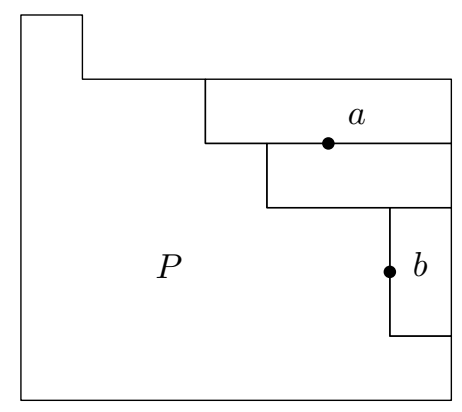

FIGURE 3. Completing L-shape from a staircase polygon $P$. Guard $a$ covers two new rooms and guard $b$ covers new room and a room from the staircase polygon.

We further improve the upper bound for staircase polygons.

THEOREM 2.1. An orthogonal staircase with $n$ rooms and $r$ reflex vertices can be guarded with $\lceil(n+\lfloor r / 2\rfloor) / 2\rceil$ guards. 


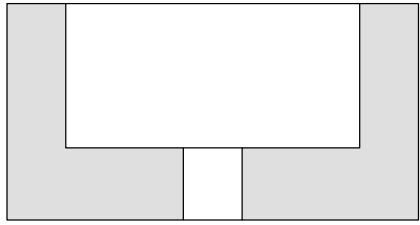

(a)

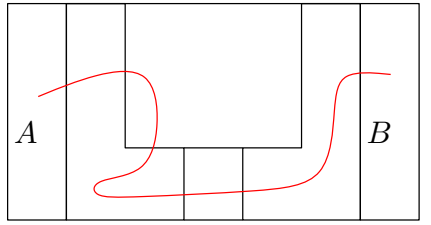

(c)

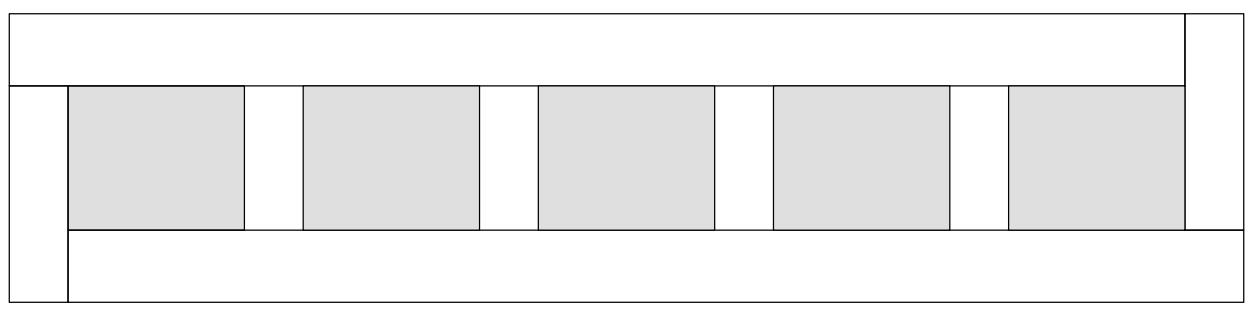

(b)

FIGURE 1. (a) Rectangle $R$ using two L-shaped polygons. (b) The construction of Lemma 1. (c) A path from $A$ to $B$ visits one of the L-shaped polygons only one time.

Proof. Proof. We prove by induction by $r$, the number of reflex vertices of the orthogonal staircase.

Base case. If $r=1$ then this is the result from Lemma 1 .

Induction step. Suppose that the claim holds for staircases with $r-1$ reflex vertices. We prove it for a staircase $P$ with $r$ reflex vertices.

Suppose that $r$ is even and $r=2 k$. We add a new room in order to form a staircase with $n^{\prime}=n+1$ rooms and $r^{\prime}=r-1=2 k-1$ reflex vertices. By induction hypothesis, the new staircase can be guarded by $\left\lceil\left(n^{\prime}+\right.\right.$ $\left.\left.\left\lfloor r^{\prime} / 2\right\rfloor\right) / 2\right\rceil=\lceil(n+1+(k-1)) / 2\rceil=\lceil(n+\lfloor r / 2\rfloor) / 2\rceil$ guards. The claim follows if we move one guard as in Corollary 2.1.

Suppose that $r$ is odd. Consider two cases.

Case 1: $n+\lfloor r / 2\rfloor$ is odd. Let $k$ be $n+\lfloor r / 2\rfloor$. Add a new room in order to form a staircase with $r-1$ reflex vertices. Then we need $\lceil(n+1+\lfloor r / 2\rfloor) / 2\rceil=\lceil(k+1) / 2\rceil$ guards. Since $k$ is odd it is equal to $\lceil k / 2\rceil$. For guarding $P$ we move one guard as in Corollary 2.1.

Case 2: $n+\lfloor r / 2\rfloor$ is even. Take a mirror image of staircase and add $r+1$ connenting rooms. Take 5 copies of this obtained rectange and complete a new rectangle. The dual graph, including the outer face, is planar and 4-connected and by a theorem of Tutte [9] it has a Hamilton circuit. For this construction it is easy to verify that exists one copy and for this copy Hamilton circuit not entry in the top and the down rectangles. Therefore the rooms in this copy are traversed by a single path $\pi$ which is a subpath of the Hamilton circuit. The endpoints of this path are the rooms $A$ and $B$ as shown in Figure 4.

First, we will prove that exist $k=\lfloor r / 2\rfloor+1$ paths covering one of the staircases. These paths will be taken as subpaths of $\pi$. Path $\pi$ passes through $r+1$ connecting rooms $R_{1}, R_{2}, \ldots, R_{r+1}$, see Figure 4 . Without loss of generality suppose that the connecting rooms appear in this order in path $\pi$

$$
\pi=A \ldots R_{1} \ldots R_{2} \ldots R_{r+1} \ldots B .
$$

There are $r+2$ different paths between the connecting rooms and $A$ and $B$ in path $\pi$. Each of these path passes through rooms of only one of two staircases. Since $r$ is odd and $k=\lfloor r / 2\rfloor+1$, then $r=2 k-1$. We have $r+2=2 k+1$ paths. Thus one of the staircases is covered by at most $k$ paths. Suppose that the number of these paths is $m$ and their lengths are

$$
n_{1}+n_{2}+\cdots+n_{m}=n \text {. }
$$

If $m<k$ then we need at most $\left\lceil n_{i} / 2\right\rceil \leq\left(n_{i}+1\right) / 2$ guards for $i$ th path of length $n_{i}$ for any $i$. The total number of guards is at most

$$
\begin{gathered}
\left(n_{1}+1\right) / 2+\left(n_{2}+1\right) / 2+\cdots+\left(n_{m}+1\right) / 2= \\
(n+m) / 2 \leq(n+k-1) / 2=(n+\lfloor r / 2\rfloor) / 2 .
\end{gathered}
$$

The induction claim follows.

Suppose that the number of paths is $m=k$. According to the fact that $n+\lfloor r / 2\rfloor$ is even we have that $n+k-1$ is even. We now prove by contradiction that there exists $j$ such that $n_{j}$ is even.

Case 1: Suppose that $k$ is odd and all $n_{i}$ are odd. Then from $n_{1}+n_{2}+\ldots+n_{k}=n$ we obtain that $n$ is odd and $n+k-1$ is odd. Contradiction.

Case 2: Suppose that $k$ is even and all $n_{i}$ are odd. Then from $n_{1}+n_{2}+\ldots+n_{k}=n$ we obtain that $n$ is even and $n+k-1$ is odd. Contradiction.

Suppose that $n_{1}$ is even. We need $n_{1} / 2$ guards for path 1 . For $i$ th path, $i>1$, we need $\left\lceil n_{i} / 2\right\rceil \leq\left(n_{i}+1\right) / 2$. 


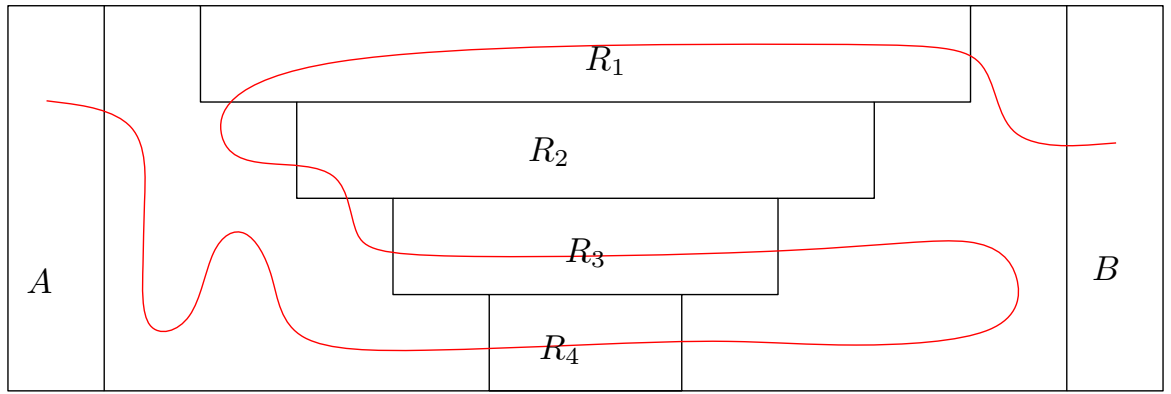

FIGURE 4. A path from $A$ to $B$.

Then we needs at most

$$
\begin{array}{r}
n_{1} / 2+\left(n_{2}+1\right) / 2+\ldots+\left(n_{k}+1\right) / 2= \\
(n+k-1) / 2=(n+\lfloor r / 2\rfloor) / 2
\end{array}
$$

guards. The theorem follows.

Now we show that, for any $r>1$, there are examples of staircase polygons that require $\lceil(n+\lfloor r / 2\rfloor) / 2\rceil$ guards to cover it.

Suppose that $r$ is odd. Consider a staircase polygon with $r$ reflex vertices and $n=(5 r+1) / 2$ rooms, see Figure 5 (a). It is easy to see that $\lceil(n+\lfloor r / 2\rfloor) / 2\rceil=$ $(3 r+1) / 2$ guards are necessary to cover this art gallery.

Now suppose that $r$ is even. Consider a staircase polygon with $r$ reflex vertices and $n=5 r / 2+1$ rooms, see Figure 5 (b). It is easy to see that $\lceil(n+\lfloor r / 2\rfloor) / 2\rceil=$ $3 r / 2+1$ guards are necessary to cover this art gallery.

\section{ORTHOGONAL POLYGONS HOLES}

WITH

Consider an orthogonal polygon $P$ with $n$ rooms, $r$ reflex vertices, and $h$ orthogonal holes. Let $B$ be the smallest rectangle containing $P$. The set $B \backslash P$ is then the union of orthogonal polygons $P_{i}^{\prime}, i=1,2, \ldots$ There are 3 types of polygons $P_{i}^{\prime}: P_{i}^{\prime}$ is of type $j(j=0,1,2)$ if the intersection of $P_{i}^{\prime}$ and the boundary of $B$ is a set of $j$ segments. Let $k_{j}, j=0,1,2$ be the number of polygons of type $j$, see Figure 6 for illustration.

TheOREM 3.1. Consider an orthogonal polygon $P$ with $n$ rooms, $r$ reflex vertices and $h$ orthogonal holes. Let $B$ be the smallest rectangle containing $P$. Suppose that $B \backslash P$ contains $k_{j}, j=0,1,2$ orthogonal polygons of type $j$.

(i) If $k_{2}>0$ then $P$ can be guarded with $\left\lceil\left(n+r-3 h-k_{1}-1\right) / 2\right\rceil$ guards.

(ii) If $k_{2}=0$ then $P$ can be guarded with $\left\lceil\left(n+r-3 h-k_{1}\right) / 2\right\rceil$ guards.

Proof. Let $r_{i}$ be the number of reflex vertices of $P$ that are also vertices of $P_{i}^{\prime}$. Let $r_{i}^{\prime}$ and $c_{i}^{\prime}$ be the number of reflex and convex vertices of $P_{i}^{\prime}$. Observe that, for any polygon $P_{i}^{\prime}$ of type $j$,

$r_{i}=c_{i}^{\prime}$, if $j=0$,

$2+r_{i}=c_{i}^{\prime}$, if $j=1$, and

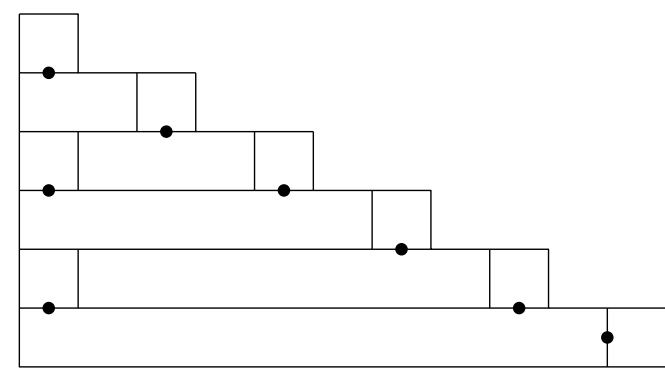

(a)

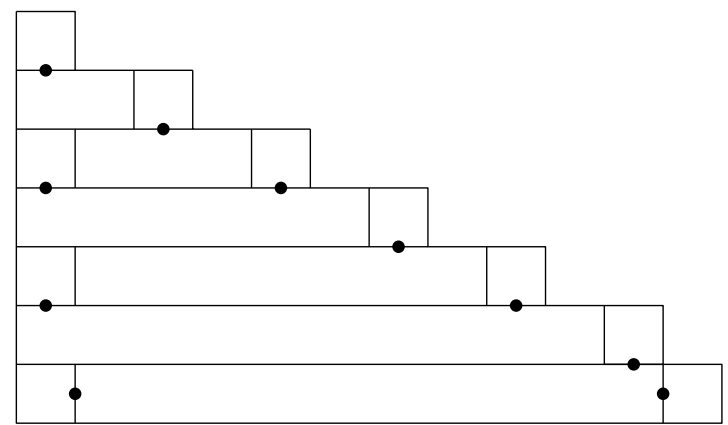

(b)

FIGURE 5. (a) Staircase with $r=5$ reflex vertices and $n=13$ rooms. It requires $(3 r+1) / 2=8$ guards. (b) Staircase with $r=6$ reflex vertices and $n=16$ rooms. It requires $3 r / 2+1=10$ guards.

$3+r_{i}=c_{i}^{\prime}$, if $j=2$.

It is easy to verify that any $P_{i}^{\prime}$ can be partitioned into $n_{i}^{\prime}=r_{i}^{\prime}+1$ rooms. Since $P_{i}^{\prime}$ is an orthogonal polygon, $c_{i}^{\prime}=r_{i}^{\prime}+4$. Then $n_{i}^{\prime}=c_{i}^{\prime}-3$. The total number of rooms in $B \backslash P$ is

$$
\begin{aligned}
\sum n_{i}^{\prime} & =\sum\left(c_{i}^{\prime}-3\right)=\sum_{P_{i}^{\prime}-t y p e 0}\left(r_{i}-3\right) \\
& +\sum_{P_{i}^{\prime}-t y p e 1}\left(r_{i}-1\right)+\sum_{P_{i}^{\prime}-t y p e 2} r_{i} \\
& =\sum r_{i}-3 h-k_{1}=r-3 h-k_{1} .
\end{aligned}
$$

The total number of rooms in $B$ is $n+r-3 h-k_{1}$. If $k_{2}>0$ then we delete one corner room from some $P_{i}^{\prime}$. $B$ with this room removed is an L-shape. By Lemma 1 


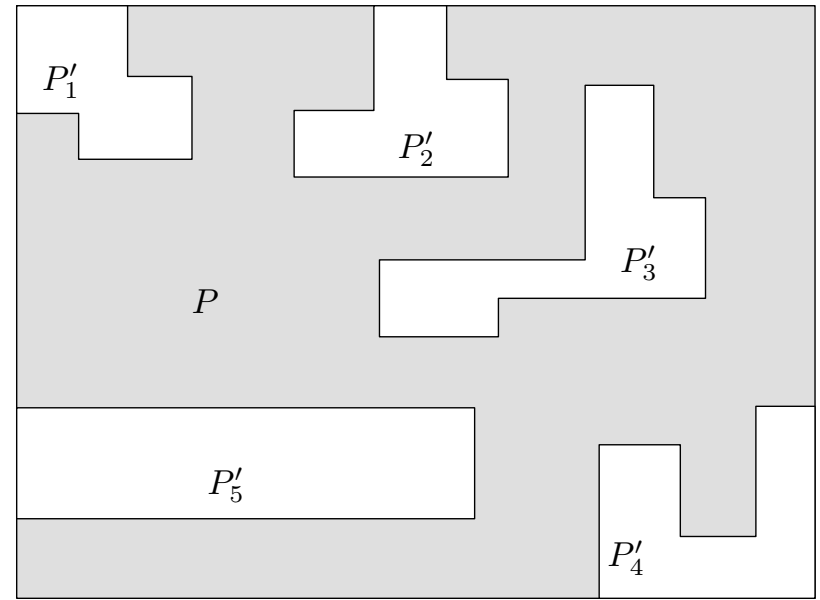

FIGURE 6. Orthogonal polygon with one hole $P_{3}^{\prime}$. The types of polygons $P_{1}^{\prime}, \ldots, P_{5}^{\prime}$ are $2,1,0,2,1$ respectively.

it can be guarded by $\left\lceil\left(n+r-3 h-k_{1}-1\right) / 2\right\rceil$ guards.

Suppose that $k_{2}=0$. Then rectangle $B$ can be guarded by $\left\lceil\left(n+r-3 h-k_{1}\right) / 2\right\rceil$ guards. The theorem follows.

Corollary 3.1. Suppose that $P$ is an orthogonal polygon without holes.

(i) If $k_{2}>0$ then $P$ can be guarded with $\left\lceil\left(n+r-k_{1}-1\right) / 2\right\rceil$ guards.

(ii) If $k_{2}=0$ then $P$ can be guarded with $\left\lceil\left(n+r-k_{1}\right) / 2\right\rceil$ guards.

Proof. It follows from Theorem 3.1 if $h=0$.

COROllary 3.2. Any orthogonal polygon with $n$ rooms, $r>0$ reflex vertices and without holes can be guarded with at most $\lceil(n+r-1) / 2\rceil$ guards.

Proof. Consider two cases of Theorem 3.1.

(i) If $k_{2}>0$ then $\left\lceil\left(n+r-k_{1}-1\right) / 2\right\rceil \leq\lceil(n+r-1) / 2\rceil$ guards suffice.

(ii) If $k_{2}=0$ then $k_{1}>0$ since $r>0$. Then $\left\lceil\left(n+r-k_{1}\right) / 2\right\rceil \leq\lceil(n+r-1) / 2\rceil$ guards suffice.

\subsection{Improvement with Chords}

We want to partition polygons $P_{i}^{\prime}$ into minimum number of rectangles. We apply the following partitioning result $[3,5,6,11]$. Let $Q$ an orthogonal polygon with $r$ reflex vertices. A chord is a line segment fully contained in (strong inside $Q$ ) that connects two reflex vertices. Let $b$ be the size of the largest set of nonintersecting chords. Ferrari et al. [3] showed that the number of rectangles in a minimum-rectangle partition of $Q$ is $r-b+1$.

We apply this result to improve our bounds in degenerate case where chords exist. Let $b_{i}$ be the size of the largest set of nonintersecting chords in polygon $P_{i}^{\prime}$ and let $b=\sum b_{i}$. The result of Theorem 3.1 can further be improved as follows.
THEOREM 3.2. Let $b$ be the size of the largest set of non-intersecting chords in $B \backslash P$.

(i) If $k_{2}>0$ then $P$ can be guarded with $\left\lceil\left(n+r-b-3 h-k_{1}-1\right) / 2\right\rceil$ guards.

(ii) If $k_{2}=0$ then $P$ can be guarded with $\left\lceil\left(n+r-b-3 h-k_{1}\right) / 2\right\rceil$ guards.

Proof. The proof is essentially the same as one of Theorem 3.1. We partition $P_{i}^{\prime}$ into $n_{i}^{\prime}=r_{i}^{\prime}-b_{i}+1$ rooms using the result of Ferrari et al. [3].

\section{DISCUSSION}

In this paper we addressed the problem of guarding orthogonal galleries with rectangular rooms. We obtained exact bounds for a subclass of orthogonal polygons (staircase polygons) and then improved the previous bound [2] for arbitrary orthogonal polygons.

We plan to continue the work on guarding orthogonal galleries with rectangular rooms. In particular we are interested in developing efficient algorithms for positioning guards. We also want to study other variations of this problem that have real life applications.

\section{REFERENCES}

[1] V. Chvátal. A combinatorial theorem in plane geometry. J. Combin. Theory Ser. B, 18, 1975.

[2] J. Czyzowicz, E. Rivera-Campo, N. Santoro, J. Urrutia, and J. Zaks. Guarding rectangular art galleries. Discrete Applied Mathematics, 50:149-157, 1994.

[3] L. A. Ferrari, P. V. Sankar, and J. Sklansky. Minimal rectangular partitions of digitized blobs. Computer Vision, Graphics, and Image Processing, 28(1):58-71, 1984.

[4] J. Kahn, M. M. Klawe, and D. Kleitman. Traditional galleries require fewer watchmen. SIAM J. Algebraic Discrete Methods, 4:194-206, 1983.

[5] W. Lipski, E. Lodi, F. Luccio, C. Mugnai, , and L. Pagli. On two-dimensional data organization ii. Fundamenta Informaticae, 2:245260, 1979.

[6] T. Ohtsuki. Minimum dissection of rectilinear regions. In Proceedings of IEEE Symposium on Circuits and Systems, pages 1210-1213, 1982.

[7] J. O'Rourke. An alternative proof of the rectilinear art gallery theorem. J. Geom., 21:118-130, 1983.

[8] J. O'Rourke. Art Gallery Theorems and Algorithms. The International Series of Monographs on Computer Science. Oxford University Press, New York, NY, 1987.

[9] W. Tutte. A theorem on planar graphs. Trans. Amer. Math. Soc., 82:99-116, 1956.

[10] J. Urrutia. Art gallery and illumination problems. In Jörg-Rüdiger Sack and Jorge Urrutia, editors, Handbook of Computational Geometry, pages 973-1027. North-Holland, 2000.

[11] C. Worman and J. M. Keil. Polygon decomposition and the orthogonal art gallery problem. International Journal of Computational Geometry and Applications, 17(2):1-34, 2007. 